\title{
Equal Sums of Biquadrates
}

\section{By L. J. Lander and T. R. Parkin}

Solutions of the Diophantine equation $A^{4}+B^{4}=C^{4}+D^{4}$ in least integers have been obtained by several authors [1]-[4]. The term primitive denotes a solution for which unity is the greatest common divisor of all the numbers $A, B, C, D$. A CDC 3200 computer program was written to search exhaustively for primitives, yielding the 31 solutions listed in Table I. The range covered is $A^{4}+B^{4}<7.885 \times 10^{15}$. The first six solutions were identified in [3] and the seventh is cited in [1].

Euler [1] gave a two-parameter algebraic solution which can be written

$$
A=f(x, y) B=f(y,-x) C=f(-x, y) D=f(y, x)
$$

TABLE I

\begin{tabular}{|c|c|c|c|c|c|}
\hline$i$ & $N_{\imath}$ & $A$ & $B$ & $C$ & $D$ \\
\hline 1 & $635,318,657$ & 158 & 59 & 134 & 133 \\
\hline 2 & $3,262,811,042$ & 239 & 7 & 227 & 157 \\
\hline 3 & $8,657,437,697$ & 292 & 193 & 257 & 256 \\
\hline 4 & $68,899,596,497$ & 502 & 271 & 497 & 298 \\
\hline 5 & $86,409,838,577$ & 542 & 103 & 514 & 359 \\
\hline 6 & $160,961,094,577$ & 631 & 222 & 558 & 503 \\
\hline 7 & $2,094,447,251,857$ & 1203 & 76 & 1176 & 653 \\
\hline 8 & $4,231,525,221,377$ & 1381 & 878 & 1342 & 997 \\
\hline 9 & $26,033,514,998,417$ & 2189 & 1324 & 1997 & 1784 \\
\hline 10 & $37,860,330,087,137$ & 2461 & 1042 & 2141 & 2026 \\
\hline 11 & $61,206,381,799,697$ & 2797 & 248 & 2524 & 2131 \\
\hline 12 & $76,773,963,505,537$ & 2949 & 1034 & 2854 & 1797 \\
\hline 13 & $109,737,827,061,041$ & 3190 & 1577 & 2986 & 2345 \\
\hline 14 & $155,974,778,565,937$ & 3494 & 1623 & 3351 & 2338 \\
\hline 15 & $156,700,232,476,402$ & 3537 & 661 & 3147 & 2767 \\
\hline 16 & $621,194,785,437,217$ & 4883 & 2694 & 4397 & 3966 \\
\hline 17 & $652,057,426,144,337$ & 5053 & 604 & 5048 & 1283 \\
\hline 18 & $680,914,892,583,617$ & 4849 & 3364 & 4303 & 4288 \\
\hline 19 & $1,438,141,494,155,441$ & 6140 & 2027 & 5461 & 4840 \\
\hline 20 & $1,919,423,464,573,697$ & 6619 & 274 & 5942 & 5093 \\
\hline 21 & $2,089,568,089,060,657$ & 6761 & 498 & 6057 & $\tilde{5} 222$ \\
\hline 22 & $2,105,144,161,376,801$ & 6730 & 2707 & 6701 & 3070 \\
\hline 23 & $3,263,864,585,622,562$ & 7557 & 1259 & 7269 & 4661 \\
\hline 24 & $4,063,780,581,008,977$ & 7604 & 5181 & 7037 & 6336 \\
\hline 25 & $6,315,669,699,408,737$ & 8912 & 1657 & 7559 & 7432 \\
\hline 26 & $6,884,827,518,602,786$ & 9109 & 635 & 9065 & 3391 \\
\hline 27 & $7,191,538,859,126,257$ & 9018 & 4903 & 8409 & 6842 \\
\hline 28 & $7,331,928,977,565,937$ & 9253 & 1104 & 8972 & 5403 \\
\hline 29 & $7,362,748,995,747,617$ & 9043 & 5098 & 8531 & 6742 \\
\hline 30 & $7,446,891,977,980,337$ & 9289 & 1142 & 9097 & 4946 \\
\hline 31 & $7,532,132,844,821,777$ & 9316 & 173 & 9197 & 4408 \\
\hline
\end{tabular}

Received September 6, 1965. 
where $f(x, y)=2 x^{7}-x^{6} y+20 x^{5} y^{2}+17 x^{4} y^{3}+2 x^{3} y^{4}+17 x^{2} y^{5}+8 x y^{6}-y^{7}$. The primitives corresponding to $i=1,7$ and 14 of Table I are special cases of this solution for the arguments $(x, y)=(3,1),(2,1)$, and $(5,1)$ respectively.

The computer program generated all values of $N=A^{4}+B^{4}$ in ascending order by controlling the advance of a series of pairs of values $A, B$ while monitoring $N$ for coincidences. To advance from a given starting value of $N$, all integers $A$ for which $N / 2 \leqq A^{4} \leqq N$ were considered; for each $A$ a corresponding $B$ was chosen as the largest integer in the range $0 \leqq B \leqq A$ for which $A^{4}+B^{4} \leqq N$. Then the smallest value $A_{1}{ }^{4}+B_{1}{ }^{4}$ in the set was found and $B_{1}$ was advanced if $B_{1}<A_{1}$, the lower limit on $A$ was advanced if $B_{1}=A_{1}$, and the upper limit on $A$ was advanced if $B_{1}=0$.

A similar computer program generated sums of three biquadrates $A^{4}+B^{4}+C^{4}$ in ascending order and found the least triple coincidence to be

$$
811,538=29^{4}+17^{4}+12^{4}=28^{4}+21^{4}+7^{4}=27^{4}+23^{4}+4^{4} .
$$

It was discovered quite by chance (using a computer program which decomposes numbers into sums of biquadrates by trial) that for the $N_{i}$ of Table I

$N_{1}+1=635,318,658=159^{4}+58^{4}+1^{4}=134^{4}+133^{4}+1^{4}=154^{4}+83^{4}+71^{4}$ is the sum of three biquadrates in three distinct ways, and that

$$
N_{3}+1=8,657,437,698
$$

is the sum of three biquadrates in five distinct ways, namely

$$
(296,157,139)^{4}=(293,184,109)^{4}=(292,193,1)^{4}=(271,239,32)^{4}=(257,256,1)^{4} .
$$

1. L. E. Dickson, History of the Theory of Numbers, Vol. 2, pp. 644-647, Publication No. 256, Carnegie Institution of Washington, Washington, I). C., 1920; reprint, Stechert, New York, 1934.

2. C. S. Ogilvy, Tomorrow's Math, Oxford, 1962, p. 94.

3. J. LeEch, "Some solutions of IDiophantine equations," Proc. Cambridge Philos. Soc., v. 53,1957, pp. $78-780$. MR 19, 837.

4. R.SpIRA, Math. Comp., v.17, 1963, p. 306. 